\title{
Desafíos y posibilidades de la investigación cualitativa en Psicología: problematizaciones necesarias
}

\author{
Challenges and possibilities of qualitative \\ research in Psychology: necessary \\ problematizations
}

\section{Desafios e possibilidades da pesquisa qualitativa em Psicologia: problematizações necessárias}

\author{
Sabrina Daiana Cúnico \\ Pontifícia Universidade Católica do Rio Grande do Sul, Brasil \\ Adolfo Pizzinato \\ Universidade Federal do Rio Grande do Sul, Brasil \\ Marlene Neves Strey \\ Pontifícia Universidade Católica do Rio Grande do Sul, Brasil \\ Almudena Garcia Manso \\ Universidad Rey Juan Carlos, España
}

Autor referente: sabrinacunico@yahoo.com.br

Historia Editorial:

Recibido: 14/06/2017

Aceptado: 23/03/2018

RESUMEN

Relatar los procedimientos y desafíos de un estudio, más que una simple perfeccionamiento y fortalecimiento de diferentes modos de hacer formalidad, se configura como una investigación. De esta forma, este importante forma de contribuir para el artículo buscó describir el método 
utilizado en una investigación cuyo objetivo fue comprender los significados dados a la paternidad de los hombres que están privados de libertad, además de discutir los desafíos y posibilidades de un trabajo de campo realizado en una institución penitenciaria masculina. Entre las innumerables posibilidades de elección, la metodología cualitativa de orientación etnográfica sostuvo la investigación aquí descripta, una vez que fue considerada una herramienta importante para deshacer padrones, conceptos e ideas que podamos llegar a tener sobre como las personas conducen y viven sus propias vidas.

Palabras clave: Prisión; Paternidad; Investigación Cualitativa; Metodología

\section{ABSTRACT}

Reporting the procedures and challenges of a study, rather than a simple formality, is an important way to contribute to the improvement and strengthening of different manners of doing research. Thus, this article sought to describe the method used in a research which aimed to understand the meanings attributed to paternity by men who are deprived of freedom, as well as discuss the challenges and possibilities of fieldwork conducted in a male-only prison institution. Among the many possibilities of choice, the qualitative methodology of ethnographic orientation supported the research described here, since it was considered an important tool for us to deconstruct potential social patterns, conceptions and ideas about how people lead and live their lives.

Keywords: Prison; Fatherhood; Qualitative research; Methodology

\section{RESUMO}

Relatar os procedimentos e desafios de um estudo, mais do que uma simples formalidade, configura-se como uma importante forma de contribuir para o aprimoramento e fortalecimento de diferentes modos de se fazer pesquisa. Deste modo, este artigo buscou descrever o método utilizado em uma pesquisa que teve por objetivo compreender os significados atribuídos à paternidade por homens que estão privados de liberdade, além de discutir os desafios e possibilidades de um trabalho de campo realizado em uma instituição prisional masculina. Dentre as inúmeras possibilidades de escolha, a metodologia qualitativa de orientação etnográfica sustentou a pesquisa aqui descrita, uma vez que foi considerada uma ferramenta importante para desconstruirmos padrões, concepções e ideias que possamos vir a ter sobre como as pessoas conduzem e vivem as suas vidas.

Palavras-Chaves: Prisão; Paternidade; Pesquisa qualitativa; Metodologia

I objetivo de este texto es discutir sobre el método construido en una
investigación que tuvo la intención de comprender, por medio de una 
perspectiva de género, los significados atribuidos a la paternidad por hombres que están privados de libertad, además de discutir los retos y posibilidades de un trabajo de campo cualitativo en Psicología, realizado en una institución carcelaria masculina. Las inúmeras posibilidades de cuestionamientos en investigación académica permiten el uso de metodologías diversas, las cuales deben estar consonantes a la problemática a ser desarrollada. Para ilustrar esa discusión, presentase una investigación que ha buscado saber la forma como los hombres que están en pena privativa de libertad comprenden y significan su condición de padres. Ese es un problema de investigación que, ciertamente, nos remite a una dimensión no cuantificable y que invita a una literatura aun difusa en muchos aspectos.

Si tomamos como base las direcciones del método científico, las cuales se sustentan en nociones de una "objetividad científica descorporificada" (Haraway, 1995 p. 9), percibimos que las posturas más sensibles y flexibles en el trabajo de campo acaban por ser obstaculizadas por esa sistematización de trabajo. De hecho, las investigaciones de tradición positivista siempre han dado prioridad para una escoja de métodos y técnicas de investigación que buscasen excluir la subjetividad del investigador a lo largo de la conducción del estudio. El objetivo era garantizar la objetividad de modo a tornar universales y generalizar los resultados encontrados.

Sin embargo, es desde las críticas hechas por el campo de la sociología y de la antropología a los parámetros "universales" de cientificidad que, irónicamente, la psicología también ha pasado a cuestionar y reconocer la inevitabilidad de la presencia de sentimientos, emociones e intuiciones del investigador y de todos los involucrados en la pesquisa como algo del propio proceso de investigación en el área. Al reconocer la subjetividad como algo inevitable, las investigaciones de tradición hermenéutica y comprensiva no buscan controlar esa subjetividad como pretenden las investigaciones de orientación positivista. En contrario, objetivan ponerla a servicio de 
la producción del conocimiento (Soledad Manrique, Di Matteo y Sanchez Troussel, 2016).

Sin embargo, la hegemonía de la visión de ciencia que se sostiene en el modelo de las ciencias naturales o exactas, subvierte a un rol secundario las áreas que utilizan un abordaje metodológico de fundamentación epistemológica relativista. En tal contexto, la Psicología, aunque sea una ciencia en que distintos abordajes reivindican metodologías diversas, demarcando también diferencias epistemológicas importantes (Silvino, 2007), busca frecuentemente consolidar su lugar en el campo mayoritario de las ciencias de la naturaleza.

De acuerdo con Smedslund (2015), sin embargo, existen un mínimo de cuatro razones y limitaciones para que la Psicología abandone el actual paradigma epistemológico que busca desarrollar una ciencia empírica en el sentido clásico, por ejemplo, formulando y testando hipótesis generales. Ellas son: la irreversibilidad, el número infinito de determinantes, el supuesto empirismo y la interacción social.

La irreversibilidad es una limitación en la medida en que se considera que es una de las características principales de los procesos psicológicos. Es decir, cada experiencia cambia una persona de una manera que no puede ser completamente deshecha. Así, diferentemente de las descubiertas en las ciencias naturales, los datos de la investigación psicológica son, en principio, históricos y temporales, una vez que no son replicables bajo las mismas condiciones iniciales. Asimismo, como consecuencia directa de la irreversibilidad tenemos el hecho de que la experiencia es infinitamente variada y, muchas veces, fortuita, lo que hace con que las personas perciban el mundo y actúen de maneras infinitamente variables. Eso hace de la psicología mucho más compleja que la ciencia natural y la interpretación de las observaciones mucho más difíciles (Smedslund, 2015). 
Una tercera limitación es respecto a la tendencia de la investigación psicológica de producir hipótesis supuestamente empíricas. Para Smedslund (2015), una manera simples de testar ese supuesto empirismo es considerar si una negación de la hipótesis es posible y aceptable. Si la negación es inaceptable por ser considerada absurda o sin sentido, la hipótesis no es empírica, una vez que podría ter sido establecida anticipadamente. Por fin, la cuarta limitación ilustra el hecho de que muchos de los estudios en Psicología tienen por objetivo observar o investigar cómo las personas responden a los instrumentos de investigación y a las condiciones experimentales. Esas metodologías, en la busca de aproximación a las ciencias naturales, acaban dejando de lado lo que es central y esencialmente humano que son las interacciones entre las personas. Tales interacciones son, muchas veces, bien explotadas en investigaciones cualitativas, las cuales se alejaren del paradigma dominante, en que criterios de objetividad y generalidad son cruciales, no son consideradas.

A partir de esas consideraciones, la cuestión que se pones es: ¿cómo producir un conocimiento crítico, que vise a la transformación de las realidades sociales y que sea, verdaderamente, construido conjuntamente entre investigador(a) e investigados(as)? (Barcinski, 2014). ¿Cómo pensar en otras formas de hacerse investigación en Psicología, para más allá del paradigma neutro positivista?

El primer paso, tal vez, es reconocer la implicación social y política del investigador (a) en el proceso de investigación, así como comprender el conocimiento producido como situado y corporificado. Nos parece que asumir el posicionamiento de que los saberes son históricamente localizados y son siempre de carácter parcial, es reconocer el objeto del conocimiento como actor y agente, no como mero recurso en la busca de la pretensa ilusión de un conocimiento universal y neutral (Haraway, 1995). Para tanto, es necesario que el (la) investigador (a) tenga consciencia de sus propias 
suposiciones y valores, dado la influencia de esas creencias en sus teorizaciones (Harding, 1993).

En ese contexto, el análisis de la implicación asume mucha importancia, puesto que permite que el(la) investigador(a) trabaje con la relación que él (ella) propio(a) establece con el objeto de conocimiento, trayendo para el campo de análisis sentimientos y percepciones subjetivas como elementos de análisis a ser trabajados y no camuflados o escondidos (Fortuna, Mesquita, Matumoto y Monceau, 2016).

Más allá de una mera elección de abordaje metodológico, el análisis de implicación puede servir como un dispositivo para problematizar los lugares instituidos de saber/poder que ocupamos en la conducción de un proceso investigativo y su impacto en la construcción de la investigación, desde las elecciones teóricas hasta la interpretación de los resultados, pasando por la emergencia del problema y la elección de las estrategias metodológicas. Cuestionar nuestras prácticas profesionales es reconocer el carácter político de toda intervención, puesto que coloca en jeque el lugar que ocupamos y problematiza nuestras prácticas mientras productoras de verdades vistas como absolutas, universales y eternas (Coimbra y Nascimento, 2008).

En ese panorama que refleja la necesidad de realización de estudios críticos y que reconozcan la dimensión política de la investigación, las epistemologías feministas parecen inscribirse como una elección ideal para la interpretación de las informaciones recaudadas en campo. Las epistemologías feministas denuncian el carácter androcéntrico de la ciencia positivista, mientras ilustran la invisibilidad de las mujeres en la ciencia, así como señalan para el hecho de las mujeres - mientras objetos de la ciencia - ter sido definidas como inferiores biológicamente a los hombres y, por eso, inaptas a ella.

En ese sentido, Giffin (2006) afirma que la búsqueda tradicional por la objetividad y neutralidad del sujeto del conocimiento involucra, invariablemente, una negación de 
acción de los individuos en el campo social. Así, "mientras los sujetos del cotidiano no son reconocidos como partícipes de la producción del conocimiento y de la construcción de la realidad social, el sujeto científico seria 'neutralizado' por medio de las reglas científicas (p. 637). Es por eso que la investigación feminista atribuye énfasis especial para la relación desigual de poder entre o(a) investigador(a) y los(as) participantes, así como para el impacto de la investigación en los(as) participantes (Narvaz y Koller, 2006), exigiendo que el investigador actúe como un sujeto ético y político y asuma una postura de auto reflexión (Giffin, 2006).

Considerando esa definición, es posible afirmar que la ciencia - en esa perspectiva feminista - supone género. Eso equivale a decir que existen diversas maneras de producirse conocimiento y que hombres y mujeres hacen ciencia de formas diferenciadas (Narvaz y Koller, 2006). Esa cuestión se torna especialmente relevante cuando se tiene por base el escenario en que la investigación aquí citada se ha desarrollado: una cárcel masculina.

\section{Contextualizando el escenario y la metodología de la investigación}

Con el crecimiento exponencial de la población encarcelada en Brasil y en el mundo (Moura y Ribeiro, 2015; Techera, Garibotto y Urreta, 2012), diversos estudios han sido hechos con esa población. Entre las diversas temáticas, aquellas que se relacionan con el ejercicio de la paternidad y maternidad de padres y madres que están en cumplimiento de penalidades con pérdida de libertad también están presentes. De modo general, eses estudios son mayoritariamente basados en métodos cuantitativos de análisis y menor uso de las metodologías cualitativas (Easterling y Johnson, 2015). La investigación cualitativa puede ser definida como una forma de hacer pesquisa que se detiene prioritariamente en análisis de los significados subjetivos cotidianos y de la experiencia singular (Flick, 2009). El eje central del paradigma cualitativo está ubicado en la capacidad humana de responder selectivamente a los estímulos externos. La 
manera como cada persona responderá a tales estímulos es influenciada directamente por el modo como interpreta y comprende las más diversas situaciones y hechos (Queiroz, Vall, Souza y Vieira, 2007).

Asimismo, las investigaciones cualitativas, por dar voz a los participantes, ofrecen detalles y aspectos que son, muchas veces, inalcanzables por los protocolos cuantitativos. En contrario a lo que ocurre en una metodología de análisis cuantitativa, que exige que el investigador sea esencialmente un analista externo de los datos colectados, en la investigación cualitativa, el investigador está involucrado en un proceso social fluido y colaborativo (Easterling y Johnson, 2015).

Esas fueron las cuestiones que movilizaron la elección de un diseño cualitativo como más apropiado para comprender cuales son los significados atribuido a la paternidad por hombres privados de libertad. A lo largo de la construcción del trabajo metodológico, nos ha parecido obvio que técnicas estandarizadas y cuantitativas de análisis no serían las más indicadas para conseguir atingir el objetivo propuesto. Sin embargo, también era necesario ir más allá de apenas entrevistar o mantener contactos con unos pocos hombres, padres, y que estuviesen privados de libertad. Esos contactos esporádicos, descontextualizados de la especificidad de una institución como la prisión, tal vez apenas nos revelase un carácter estático respecto a la experiencia de la paternidad, lo que absolutamente no le es intrínseco.

En ese sentido, era necesario transponer los estudios tradicionales de Goffman y Foucault respecto de la cárcel - estudios hechos para realidades de cárceles distintas de la realidad brasileña - y conocer las particularidades de la institución en que la investigación seria realizada. Así, el estudio fue conducido en una prisión masculina ubicada en una ciudad del sur de Brasil, con un contingente de más de cuatro mil hombres. El objetivo de ese trabajo de campo fue el de aproximación a la cárcel, aunque desde una posición extremamente privilegiada y poder retratar esa realidad. 
El trabajo fue realizado en dos momentos distintos. El primer, de orientación etnográfica, ha involucrado observaciones participantes y el segundo ha consistido en la realización de entrevistas semiestructuradas. Las observaciones fueron realizadas en un período de tres meses en la Sala de Visitas - anteriormente llamada de Sala de Revista y local donde los familiares pasan por los procedimientos de seguridad y cacheo antes de entrar en la institución - y también en las fiestas del Día del Niño y Navidad, organizadas por la administración de la prisión.

El interese en la aproximación de los familiares que visitan alguna persona encarcelada, en especial las mujeres que llevan los hijos, estuvo íntimamente relacionado con la preocupación de que la temática no estuviera distante de una perspectiva relacional entre los géneros. Así, escuchar también lo que tenían que decir las mujeres respecto a la paternidad en ese contexto iba al encuentro de una visión no esencialista de la experiencia de ser padre.

Iniciar el trabajo de campo por una metodología de orientación etnográfica fue la forma escogida para conocer aquello que es esperado socialmente, lo que es legislado e instituido y lo que realmente acontece en la práctica y/o en el cotidiano de las personas. La aproximación y la involucración que son posibilitadas por el ejercicio etnográfico ilustran la mirada de "cerca" y de "dentro", yendo más allá de meras suposiciones sobre la realidad que está siendo estudiada.

En otros términos, se ha buscado dislocar los sujetos involucrados de una posición de meros proveedores de datos para otra en que son protagonistas en el proceso de investigación (Chaves y Paulon, 2015). Todo el trabajo de campo fue acompañado de registros en un diario. Tales diarios comprenden el modo como fueron realizados los contactos con los familiares, así como la forma como la investigadora fue recibida por ellos (Duarte, 2002). 
El segundo momento de la investigación ha sido la realización de entrevistas con doce hombres que estaban cumpliendo pena de privación de libertad y que eran padres. La elección de la entrevista se ha dado en función de ser un instrumento adecuado para aprehender e interpretar la realidad por medio del punto de vista de los actores sociales (Poupart, 2008). La elaboración de las cuestiones de la entrevista intento dejar de lado las preguntas cerradas del tipo "¿por qué?" , priorizando aquellas que se proponían a comprender como era la experiencia de vida de los participantes y su relación con la paternidad.

La selección de los entrevistados se ha dado mediante envío de los criterios de inclusión para el lugar responsable por la orientación de las investigaciones en la prisión. Con los criterios en manos - que eran apenas que los padres estuviesen reclusos a más de seis meses, sin restricciones respectos al delito/crimen cometido la administración ha solicitado a un profesional que mantenía contacto directo con los presos para que hiciese las invitaciones para participar en la investigación. El tiempo mínimo de reclusión fue pensado en función para que los significados atribuidos a la paternidad estuviesen más consolidados debido al alejamiento entre padre e hijos.

El número de sujetos participantes fue limitado por el criterio de saturación, (concepto introducido por Glaser y Strauss 1967 en Francis et al., 2010), que se refiere a el punto en que nuevas informaciones no desarrollan aspectos de la categoría conceptual. Es hecho que, aunque ampliamente utilizado en investigaciones de cariz cualitativo, aun hacen falta estudios que documenten el proceso involucrado en la búsqueda por el punto de saturación, lo que genera inúmeras críticas por parte de quienes no son familiarizados con ese tipo de pesquisa.

En función de eso, Guest, Bunce y Johnson (2006) desarrollaron un estudio con el objetivo de identificar cuantas entrevistas son necesarias para terse un sentido confiable de que la saturación de los datos fue, de hecho, alcanzada. De acuerdo con 
esos autores, en investigaciones semejantes a esa - en que entrevistas semiestructuradas son realizadas con un grupo relativamente homogéneo para comprender percepciones comunes - cerca de doce entrevistas son suficientes para alcanzar la saturación. Un criterio cuantitativo del concepto, pero que busca dar cuenta de la demanda de explicaciones relacionadas al punto de saturación de los datos.

\section{La investigación en una institución penitenciaria}

Son muchos los desafíos presentados en los inicios de la investigación en una institución de control y vigilancia, como lo es la prisión. Uno de los primeros desafíos es el concerniente a la aprobación de la investigación por las entidades competentes. Como toda investigación que incluye personas humanas, es necesario la aprobación de un estudio por parte de un Comité de Ética (CEP), quien evalúa si la investigación respeta los aspectos éticos, si existen riesgos para los informantes o si se prevé el silencio y la confidencialidad de las identidades de los sujetos a estudio (Resolución $510 / 2016)$.

En investigaciones que incluyen hombres y mujeres presos, sin embargo, los investigadores también necesitan la aprobación del órgano responsable de la administración de los presidios del Estado en concreto. En el caso de esta investigación, al mismo tiempo que en el CEP se exigía la aprobación de la Superintendencia para que diese su parecer, la Superintendencia exigía la aprobación del CEP antes de iniciar la evaluación del estudio. Fue necesario que la investigadora interviniese y negociara con las dos instancias con el fin de poder obtener ambas aprobaciones. Lo que hizo que el comienzo a la recolección de los datos se llegara a postergar.

Además, de acuerdo con la resolución 510 del Conselho Nacional de Saúde (2016), los individuos privados de libertad deben firmar los Términos de Asentimiento (AT), documento elaborado para los menores de edad o para las personas incapacitadas de 
manera temporal den o no su consentimiento. Así como el Término de Consentimiento Libre y Esclarecido (TCLE), o TA deben contener el objetivo del estudio, la metodología empleada, bien como garantías de que las identidades de los participantes sean mantenidas en silencio. La equiparación de la vulnerabilidad con la necesidad de protección especial de los seres humanos sugiere que prisioneros, así como los niños, carecen de capacidad y no están en posición de decidir por sí mismos (Arditti, 2015).

Obviamente, la obligatoriedad de la firma del TA por parte de los individuos privados de libertad tiene por objetivo el de proteger a los presos de cualquier coacción para participar en la investigación, asegurando sus derechos de consentir o no su participación (Byrne, 2005). Los ejemplos más comunes de esta coacción incluyen la presión directa hecha por los profesionales de la prisión y/o su participación será motivo de castigo (Moser et al., 2004). Aún, es preciso considerar que la firma de un documento en el contexto presidiario -sea este de cualquier tipo- puede levantar y reforzar barreras que son inherentes al estudio con poblaciones institucionalizadas, perpetuando su silencio.

Además, es posible cuestionar cuánto de efectiva y garante es la firma del TA y si refleja la voluntad real del propio sujeto en la participación de la investigación. Una vez que, de acuerdo con Moser et al. (2004) las principales razones de los presos para participar en los estudios externos incluían evitar el tedio, encontrar personas diferentes y mostrarse cooperativos, con la esperanza de que serán tratados mejor dentro del presidio. En este panorama, cuestionamos cuánto hay de cierto en la libertad de actuación de los presos a la hora de consentir su participación en las investigaciones en el contexto del presidio. Ya que se puede esperar que los presos considerados "recuperables" se muestren más solícitos y quieran ayudar a la 
sociedad. Es por esta razón que algunos autores (Moser, et al., 2004) entienden que el ambiente del presidio es inherentemente coercitivo.

Esta situación se tornó en particularmente relevante en la investigación aquí citada, en la medida en que todos los sujetos privados de libertad fueron indicados por la prisión. Por cierto que, en una cantidad de más de cuatro mil hombres, escoger aquellos que pueden ser informantes clave potenciales no es algo fácil para un investigador que no trabaje en la institución y, por tanto, no conoce a sus internos. Sin embargo, considerando que los individuos indicados eran, en su mayoría, participantes de un proyecto de desintoxicación - cuya elección sobre quién puede integrar el proyecto, es responsabilidad del propio presidio- y/o presos que trabajaban en la institución, lo que es de suponer que la elección de estos sujetos no fue en ningún momento aleatoria, ni fiel a la diversidad del universo muestral, que sería la totalidad de los presos que son padres y que están en dicho presidio.

Conforme ya mencionado por otros autores (Arditti, 2015, Byrne, 2005), realizar investigaciones en un contexto como lo es una prisión requiere que el investigador tenga en cuanta, ciertas adaptaciones. En el presidio, la idea de que las entrevistas deben ocurrir en un Setting adecuado, que favorezca a la comunicación entre el investigador(a) y entre el preso (a) y que deba ser garante de la confidencialidad de lo que está siendo dicho, subiendo, en ocasiones, los procedimientos de seguridad que son adoptados en la prisión. En esta investigación, por ejemplo, todas las entrevistas fueron realizadas en salas sin puerta, con policías en las proximidades y con los presos esposados con las manos atrás. Las esposas sólo fueron retiradas en el momento en el que firmaron el TA.

Evidentemente las medidas de seguridad forman parte de lo cotidiano en una prisión. En la realización de una investigación que tiene como sujetos participantes a presos, es concebible que esas medidas sean vistas como una prioridad, sin embargo, es 
necesario también reconocer cuanto las informaciones son sesgadas por estos protocolos de seguridad y pueden, inadvertidamente, silenciar a los participantes.

Estamos de acuerdo con Swauger (2011) cuando puntualiza que la relación entre el participante y el investigador es típicamente una relación jerárquica. En el caso de una investigación realizada en un espacio como lo es una prisión, la jerarquía se torna demasiado evidente si consideramos el grado de instrucción y de responsabilidad por la demarcación de los días y los horarios de las entrevistas, por ejemplo. Es preciso, entonces, que el investigador tenga claro el lugar que ocupa en lo que respecta a su relación con los sujetos a estudio. Mantenerse consciente de que su identidad no significa, necesariamente, poder equilibrar con los participantes, ayuda más al establecimiento de una relación sólida y honesta.

¿Cómo garantizar, sin embargo, que la entrevista sea un momento de encuentro donde el investigado(a) y el investigador (a) están desarrollando el contenido a estudio, mientras el sujeto a estudio está encadenado con las manos hacia atrás? ¿O, cómo se espera que una persona privada de libertad hable de manera libre y espontánea sobre su vida, estando en una sala sin puerta, lo cual no garantiza en absoluto la confidencialidad de sus datos y discurso? Estas cuestiones ilustran el hecho de que al proponer una investigación en la que los sujetos participantes están privados de libertad, el propio investigador necesitará encontrarse y someterse a las reglas y reglamentos impuestos por la prisión.

Es en ese sentido en el que se argumenta a favor de una mayor flexibilidad como algo esencial para poder realizar una buena base de un estudio cualitativo (Easterling y Johnson, 2015; Swauger, 2011). En la investigación aquí relatada, por ejemplo, el objeto inicial era acompañar un día de visitas de los hijos a los padres, con el fin de poder observar la interacción entre el padre y el hijo, o que es lo que hacen cuando están juntos, como es la reacción de la familia, etc. Sin embargo, como las visitas 
ocurren en las propias galerías y celdas sin la supervisión policial, la administración del presidio no permitió la entrada de la investigadora en este espacio junto a los familiares. El principal motivo alegado por la administración del presidio fue la posible amenaza que la investigadora podría representar en aquel ambiente, como fue dicho por el director:

Pienso que las mujeres de los presos no verían con buenos ojos una persona extraña allá dentro porque eso llamaría la atención de los presos. Me quedo más preocupado con la reacción de las mujeres que con la de los hombres (Diario de campo, 2015).

Todo esto nos permite observar una cuestión de vital importancia en la investigación a considerar, cómo el género atraviesa toda la investigación, lo cual hace que el rumbo que se debe tomar en la misma cambie o altere los procesos y las formas. Obviamente no podemos olvidar que esta es una investigación realizada por una mujer en una prisión masculina. Desde el primer día en el que dio comienzo la observación participante de la investigadora, se percibió cómo muchas mujeres veían a la investigadora con desconfianza. Inicialmente, esta desconfianza fue entendida como la fácil capacidad de las mujeres de los presos para reconocer cuales de las personas son nuevas, puesto que el reconocimiento y la identificación la realizan en la frecuencia del encuentro los días de visita.

Más tarde, sin embargo, la razón de la desconfianza de estas mujeres fue apareciendo. Una visitante informo que era mucho más fácil identificar que la investigadora no era una visita, pues calzaba unas botas (algo que las visitan no pueden usar), llevaba el cabello recogido (las visitas no pueden llevar ningún ornamento en el cabello u horquilla) y usaba un abrigo gris (color que no está permitida). Siendo así, era evidente saber que no se trataba de una familiar de un preso. 
La desconfianza generada por la presencia de la investigadora fue constante durante todas las observaciones, ni la misma explicación recurrente de que ella estaba ahí para hacer una investigación sobre la paternidad parecía dejarlas más tranquilas. Las aproximaciones de mujeres exaltadas y las miradas de enfrentamiento eran frecuentes. Inicialmente, la razón de estos comportamientos agresivos no parecían claros, al final las observaciones no tenían como objetivo investigar la vida y las rutinas de esas mujeres, pero sí intentar comprender cómo se hacen cargo de su familiar y cómo lidian con la situación de privación de libertad de su compañero.

En la medida que las observaciones sucedían, sin embargo, las razones por las que muchas mujeres eran confrontativas y se negaban a hablar con la investigadora sobre las dificultades y sobre los desafíos que supone la paternidad en la cárcel, resultaron ser más evidentes. A partir de los cuestionamientos que hacían, en especial aquellos que buscaban identificar cuán irrestricto era el acceso de la investigadora a las galerías y cuánto tiempo se quedaba junto a los presos, quedó claro el incómodo generado por la investigación, probablemente por el hecho de que la investigadora era mujer.

La idea de que la investigadora haya sido identificada como un riesgo para las relaciones de esas mujeres, nos muestra cómo el género es un indicador fundamental a tener en cuenta en todas las investigaciones y en concreto en esta. El hecho de ser mujer investigadora alrededor de un presidio exclusivamente para hombres influenció en los datos recolectados, así como influenció en la interacción establecida con las participantes. En efecto, la propuesta inicial era otra. Se pretendía recolectar informaciones a través de las conversaciones establecidas con las mujeres, se quería identificar a algunos hombres para realizar la entrevista en profundidad individual. Sin embargo, a pesar de las propuestas de madres de presos que intentaban programar 
encuentros románticos entre la investigadora y sus hijos, ninguna de las mujeres consultadas quiso que su compañero fuera sujeto de estudio.

La presencia de la investigadora en el espacio del presidio, tanto en la sala de visitas como en los corredores, con el fin de poder hacer la realización de las entrevistas individuales en profundidad, pareció despertar sentimientos diversos en familiares, entre los profesionales del penitenciario y entre los propios presos. Estos sentimientos orientaban el tipo de relación establecida. Por ejemplo, cuando la investigadora era vista por los profesionales del presidio como alguien que trabajaba "en favor" de los presos, tenía dificultades en su trabajo, y escuchaba comentarios como eses: “¿por qué usted no hace un trabajo con los profesionales?, nos acompañar día a día, saber las horas que dormimos, o cuanto trabajamos, ¿pero ahí nadie se interesa, verdad?" (Diario de Campo, 2015).

¿Por qué hay psicólogos que se interesan por eso aquí? Las personas que están aquí escogen vivir aquí, no van a salir de esta vida, tiene presos que no quieren salir de aquí porque ganan más dinero estando presos que sueltos (Diario de Campo, 2015).

De la misma forma, en ocasiones que era identificada como una supuesta informante para el presidio, la investigadora sentía el mismo contenido de reprobación de su trabajo por parte de los familiares de los presos, que se apartaban y evitaban conversar. Durante todos los meses en que su trabajo fue realizado, quedo patente que su papel de investigadora en aquel ambiente asumió contornos distintos. Su presencia no pasó desapercibida, ella era -en todo momento- invitada a posicionarse en un contexto que pareciese estar dicotomizado entre "nosotros" o "ellos". Como ya fue referido por Queiroz et al. (2007), la utilización de la observación participante en una investigación cualitativa "modifica la acción por parte del investigador que, al integrar un grupo que viva la realidad social, propicia interacciones, que contribuyan 
para un cambio de comportamiento do grupo observado de forma no intencional" ( $p$. 278).

En este estudio, la incursión de la investigadora en el campo profesional fue circunscrita por comentarios y rumores que la desubicaron de su posición de investigadora, y la situaban en una posición de familiar o entonces de profesional del presidio, contrapuesto a la idea de una supuesta neutralidad del investigador a lo largo del trabajo de campo. Diferente de lo que se postula la tradición racionalista y cartesiana que entiende al investigador como apartado del acto de conocer (Chaves y Paulon, 2015), en esta investigación la investigadora se configuró, evidentemente, también como herramienta de investigación.

\section{Consideraciones finales}

Este trabajo surgió de la relevancia de compartir algunas informaciones acerca de las elecciones metodológicas involucradas en una investigación que tuvo por objetivo conocer los significados atribuidos a la paternidad por hombres privados de libertad. Además de este objetivo, esta investigación pretendía problematizar y reflexionar sobre de los desafíos que una investigación de este tipo, es decir una investigación hecha dentro de un presidio masculino por una investigadora que es mujer.

Hacer la investigación en un presidio supuso el enfrentarse diversos ejes de desigualdad que interactúan entre ellos y con la realidad analizada, como son el género, la clase social y la raza. Todo esto hizo que la prisión se convirtiera en un lugar complejo, en el que cualquiera de estos ejes no pueda -0 no deba- ser considerado aisladamente. Es entonces cuando la elección por una metodología cualitativa de orientación etnográfica surge como herramienta de vital importancia a la hora de de-construir patrones, concepciones e ideas que podamos tener sobre cómo las personas conducen y viven sus vidas. La de-construcción sólo podrá concretarse a través de una apertura del propio investigador, el cual debe desprenderse de sus 
prejuicios y juicios de valor, con el fin de poder asimilar y analizar los nuevos significados y significantes.

Este trabajo no tiene como objeto argumentar a favor de la superioridad de análisis de las investigaciones cualitativas, aun tratando un tema como es el de la paternidad en prisión, cuyo abordaje se presenta más factible desde lo cualitativo. Entendemos que ambas líneas metodológicas -cuantitativa y cualitativa- son necesarias para poder dar respuestas a preguntas tales como, a considerar: cuál es el impacto del encarcelamiento en la vida de hombres y mujeres que son padres, cuáles son las consecuencias del encarcelamiento para los hijos y demás familiares y qué tipos de relaciones se establecen entre padres y madres que están en situación de prisión y sus hijos.

El informe de los procedimientos y de los desafíos de una investigación, lejos de ser una simple formalidad, se configura como una forma importante de contribuir al perfeccionamiento y fortalecimiento de diferentes modos de hacer investigación. En el caso de este estudio, entendemos que el uso del procedimiento cualitativo fue importante, no sólo para alcanzar los objetivos propuestos, sino que también fue importante para los encuestados, que tuvieron un espacio para hablar sin referirse a su implicación con la justicia.

Evidentemente, los resultados de la investigación aquí relatada se circunscriben a una determinada realidad del presidio sobre el que se hace la investigación, está investigación se lleva a cabo en un espacio de tiempo específico, una investigación que es atravesada por el género sin que por ello esto suponga ser generalizable a todas las investigaciones. Esta cuestión no menoscaba los resultados del estudio, ya que la investigación cualitativa no tiene la intención de generalizar, por ello no puede considerarse una limitación del estudio. 
Por último, el presente artículo busca cuestionar el paradigma epistemológico dominante, alentando y fomentando nuevas formas de investigación en psicología, más allá de los patrones universales, a-históricos, en los que la objetividad que se pretende conseguir se confunde con una supuesta neutralidad del investigador. El hecho de asumir la implicación del investigador en el proceso de investigación, así como la acción de los sujetos a estudio, nos parece un método que intenta asumir que la ciencia y la política son indisolubles, siendo de responsabilidad del investigador el decir si se actuará para el cambio o para el mantenimiento del status quo existente.

\section{Referencias}

Arditti, J. A. (2015). Situating vulnerability in research: implications for researcher transformation and methodological innovation. The qualitative report, 20(10), 1568-1575. Recuperado de http://nsuworks.nova.edu/tqr/vol20/iss10/2/.

Barcinski, M. (2014). O lugar da informalidade e do imprevisto na pesquisa científica: notas epistemológicas, metodológicas e éticas para o debate. Pesquisas e Práticas Psicossociais, 9(2), 278-286. Recuperado de http://www.seer.ufsj.edu.br/index.php/revista_ppp/article/view/935/738

Byrne, M. W. (2005). Conducting Research as a Visiting Scientist in a Women's Prison. Journal of Professional Nursing: Official Journal of the American Association of Colleges of Nursing, 21(4), 223-230. doi: 10.1016/j.profnurs.2005.05.001

Chaves, A. G. C. R., \& Paulon, S. M. (2015). Sobre o pesquisar uma pesquisa: notas metodológicas acerca das experimentações de uma abordagem metodológica participativa. Pesquisas e Práticas Psicossociais, 10(1), 11-23. Recuperado de http://www.ufsj.edu.br/portal2-repositorio/File/revistalapip/1_Chaves.pdf

Coimbra, C., \& Nascimento, M. L. (2008). Análise de implicações: desafiando nossas práticas de saber/poder. En A. R. R. Geisler, A. L. Abrahão \& C. M. B. Coimbra 
(Orgs.). Subjetividades, violência e direitos humanos: produzindo novos dispositivos em saúde (pp. 143-153). Niterói, RJ: EdUFF.

Conselho Nacional de Saúde (2016). Resolução do Conselho Nacional de Saúde $510 / 16$

Recuperado

de

http://conselho.saude.gov.br/resolucoes/2016/Reso510.pdf

Duarte, R. (2002). Pesquisa qualitativa: reflexões sobre o trabalho de campo. Cadernos de Pesquisa, 115, 139-154. Recuperado de http://www.scielo.br/pdf/cp/n115/a05n115

Easterling, B. A., \& Johnson, E. I. (2015). Conducting Qualitative Research on Parental Incarceration: Personal Reflections on Challenges and Contributions. The Qualitative Report, 20(10), 1568-1575. Recuperado de http://nsuworks.nova.edu/tqr/vol20/iss10/1/?utm_source=nsuworks.nova.edu\%2 Ftqr\%2Fvol20\%2Fiss10\%2F1\&utm_medium=PDF\&utm_campaign=PDFCover Pages

Flick, U. (2009). Introdução à pesquisa qualitativa. (Traductor Costa, J. E.). Porto Alegre: Artmed.

Fortuna, C. M., Mesquita, L. P., Matumoto, S., \& Monceau, G. (2016). A análise de implicação de pesquisadores em uma pesquisa-intervenção na Rede Cegonha: ferramenta da análise institucional. Cadernos de Saúde Pública, 32(9), 1-10. doi: 10.1590/0102-311X00117615.

Francis, J. J., Johnston, M., Robertson, C., Glidewell, L., Entwistle, V., Eccles, M. P., \& Grimshaw, J. M. (2010). What is an adequate sample size? Operationalising data saturation for theory-based interview studies. Psychology \& Health, 25(10), 1229-1245. doi: 10.1080/08870440903194015. 
Giffin, K. M. (2006). Produção do conhecimento em um mundo "problemático": contribuições de um feminismo dialético e relacional. Estudos Feministas, 14(3), 635-653. doi: 10.1590/S0104-026X2006000300004

Guest, G., Bunce, A., \& Johnson, L. (2006). How many interviews are enough? An experiment with data saturation and variability. Field Methods, 18(1), 59-82. Recuperado de http://journals.sagepub.com/doi/abs/10.1177/1525822X05279903.

Haraway, D. (1995). Saberes localizados: a questão da ciência para o feminismo e o privilégio da perspectiva parcial. Cadernos Pagu, 5, 7-41. Recuperado de https://periodicos.sbu.unicamp.br/ojs/index.php/cadpagu/article/view/1773.

Harding, S. (1993). A instabilidade das categorias analíticas na teoria feminista. Estudos Feministas, 1(1), 7-32. Recuperado de https://periodicos.ufsc.br/index.php/ref/article/view/15984

Moser, D. J., Arndt, S., Kanz, J. E., Benjamin, M. L., Bayless, J. D., Reese, R. L., ...Flaum, M. A. (2004). Coercion and informed consent in research involving prisoners. Comprehensive Psychiatry, 45(1), 1-9. doi: 10.1016/j.comppsych.2003.09.009.

Moura. T. W., \& Ribeiro, N. C. T. (2015). Levantamento Nacional de Informações Penitenciárias - Infopen. Departamento Penitenciário Nacional - Ministério da Justiça, 2015. Recuperado de http://www.justica.gov.br/seus-direitos/politicapenal/relatorio-depen-versao-web.pdf.

Narvaz, M. G., \& Koller, S. H. (2006). Metodologias feministas e estudos de gênero: articulando pesquisa, clínica e política. Psicologia em Estudo, 11(3), 647-654. doi: 10.1590/S1413-73722006000300021.

Poupart, J. (2008). A entrevista do tipo qualitativo: considerações epistemológicas, teóricas e metodológicas. En J. Poupart, et al. (Orgs.). A pesquisa qualitativa: 
Enfoques epistemológicos e metodológicos. (Traductor Nasser, A. C.) (pp. 215253). Petrópolis, RJ: Vozes.

Queiroz, D. T., Vall, J., Souza, A. M. A., \& Vieira, N. F. C. (2007). Observação participante na pesquisa qualitativa: conceitos e aplicações na área da saúde. Revista Enfermagem UERJ, 15(2), 276-83. Recuperado de http://www.facenf.uerj.br/v15n2/v15n2a19.pdf

Silvino, A. M. D. (2007). Epistemologia positivista: qual a sua influência hoje? Psicologia: ciência e profissão, 27(2), 276-289. Recuperado de http://pepsic.bvsalud.org/scielo.php?script=sci_arttext\&pid=S141498932007000200009.

Smedslund, J. (2015). Why Psychology Cannot be an Empirical Science. Integrative Psychological and Behavioral Science, 50(2), 1-11. doi: 10.1007/s12124-0159339-x.

Soledad Manrique, M., Di Matteo, M. F., \& Sanchez Troussel, L. (2016). Análisis de la implicación: construcción del sujeto y del objeto de investigación. Cadernos de Pesquisa, 46(162), 984-1008. doi: 10.1590/198053143559.

Swauger, M. (2011). Afterward: The ethics of risk, power, and representation. Qualitative Sociology, 34, 497-502. doi: 10.1007/s11133-011-9201-5

Techera, J., Garibotto, G. \& Urreta, A. (2012). Los "hijos de los presos": vínculo afectivo entre padres privados de liberdad y sus hijos/as. Avances de un estudio exploratorio. Ciencias Psicológicas, 6(1), 57-74. Recuperado de http://www.scielo.edu.uy/scielo.php?script=sci_arttext\&pid=S168842212012000100006 
Formato de citación

Cúnico, S., Pizzinato, A., Neves, M., Garcia, A. (2018). Desafíos y posibilidades de la investigación cualitativa en Psicología: problematizaciones necesarias. Psicología, Conocimiento y Sociedad, 8(1), 194-217. doi: http://dx.doi.org/10.26864/PCS.v8.n1.9 